zug. Es ist aber umgekehrt: einen Auszug bringt die Francofurtana. Auch bei Ivo ist die Kapitelangabe um 1 heraufzurücken: 10,135 (nicht 134). ... et pecuniam-persolvat ist übrigens erst durch Burchard hinzugefügt worden, vgl. MGH, Capit. 1, S. 362 (nicht 361) Anm. e).

München

Gerhard Schmitz

\title{
Erwiderung auf den Beitrag von Gerhard Schmitz „Die Collectio Francofurtana und Benedictus Levita. Kritische Bemerkungen zu einer neu erschienenen Edition“
}

Die Hinweise und Kritiken von Gerhard Schmitz wären sehr nützlich gewesen, wenn es sich bei unserer Ausgabe der Francofurtana um eine kritische Edition gehandelt hätte. Jedoch stellt der Band „Die Collectio Francofurtana: eine französische Decretalensammlung. Analyse beruhend auf Vorarbeiten von Walther Holtzmann $(\dagger)$ ", wie im Untertitel deutlich benannt, eine Analyse dar und eben keine Edition. Wir danken Herrn Schmitz, dass er uns die Gelegenheit bietet, erneut darlegen zu dürfen, was in der intensiv gepflegten kanonistischen Tradition die Funktion und Bedeutung einer Analyse ausmacht. Nach Walther Holtzmann besteht die Analyse in der Aufnahme von Incipit und Explicit; Versuch der Identifikation der Dekretale: Nachweis in den Regesten (soweit verzeichnet); Angabe des Druckortes, wenn noch nicht vorhanden, Edition der Dekretale; soweit vorhanden, Angaben von Bearbeitung oder Erwähnung der Dekretale in der Sekundärliteratur. Entsprechend sollten die in den Sammlungen enthaltenen vorgratianischen Texte bearbeitet werden, die ebenfalls nicht ediert werden sollten. Darüber hinaus enthält die nun von uns vorgelegte Analyse der Francofurtana zu jeder Dekretale den Nachweis des Vorkommens desselben Textes in den anderen über 60 erhaltenen Dekretalensammlungen. Von diesen Dekretalensammlungen haben neben der Francofurtana noch fünf weitere Sammlungen bisher keine Analyse erfahren. Doch nach den Vorstellungen Walther Holtzmanns, Stephan Kuttners, Charles Duggans u.a. sowie der heute Verantwortlichen des Stephan-Kuttner-Institute of Medieval Canon Law kann erst nach Abschluss dieser Grundlagenarbeit mit der übergeordneten Zielperspektive der „Regesta decretalium“ begonnen werden, nämlich den nach Incipit geordneten Regesten aller Dekretalen nach dem Decretum Gratiani bis zum Beginn des Pontifikats Papst Innocenz' III. im Jahre 1198. Die „Regesta Decretalium“ sollen zielgerichtet und arbeitstechnisch orientiert sein auf eine kritische Gesamtedition aller Dekretalen. Hierin liegt der Grund, warum die Edition einer einzelnen Sammlung keinen Sinn macht und bisher auch von Emil Friedberg, Heinrich Singer, Walther Holtzmann, Christopher und Mary Cheney nicht unternommen wurde.

Die Analyse der Francofurtana wurde in der Reihe Monumenta Iuris Canonici, Series B, vol. 9, publiziert, in der vor allem Editionen zu finden sind, darunter die von Stanley Chodorow und Charles Duggan herausgegebenen „Decretales ineditae saeculi XII“ (1982). Dies mag vielleicht erklären, warum Schmitz von uns eine Edition der Francofurtana erwartet hat statt der Analyse, die wir geboten haben. Jedoch sei nicht übersehen, dass in derselben Reihe Christopher und Ma- 
ry Cheney einen fundamentalen Band mit über zwanzig Analysen von kleineren Dekretalensammlungen vorlegten, an deren Maßstäben der Francofurtana-Band zu messen ist.

Paläographische Untersuchungen von Dekretalensammlungen stehen noch völlig aus. Von den meisten Sammlungen hat sich nur eine Handschrift erhalten, von der Francofurtana jedoch vier Manuskripte. Ein Stemma der vier Handschriften wurde von den Bearbeitern bei der Aufnahme der Incipit und Excipit in der Tat nicht angestrebt, um keine virtuelle fünfte Version zu kreieren, die für die Grundlage einer künftigen Edition unbrauchbar gewesen wäre. Die Entscheidung für Francofurtana $T$ als primäre Leithandschrift beruht darauf, dass Francofurtana $T$ (royes) aus der Entstehungsregion (Sens, Troyes oder Auxerre) stammt, keine Zusatzkapitel hat und nur wenige Glossen bringt. Im Übrigen muss berücksichtigt werden, dass die Francofurtana wegen der überleitenden Kommentierungen der Dekretalen offenbar ursprünglich ein Unterrichtswerk und keine Sammlung für kirchliche Richter wie die meisten englischen Dekretalensammlungen war. Deshalb spricht alles dafür, dass sie im Umkreis einer kanonistischen Ausbildungsstätte entstanden ist. Als eine Kathedralschule ist Sens um 1180/85 durch dort verfasste Dekretsummen belegt. Es sollte daher daran festgehalten werden, dass auch aufgrund der neueren Forschung zu Zentren in Europa im letzten Viertel des 12. Jahrhunderts die Collectio Francofurtana in der Region der Champagne kompiliert wurde, allerdings bereits frühzeitig in der Normandie, im Rhein-Mosel-Gebiet (Franken) und vielleicht auch in England Verbreitung fand ${ }^{1}$ ).

Welche Ergebnisse bringt Schmitz nun auf seinem Gebiet der Studien zur Collectio Capitularium Benedicti Levitae? Seine Kritik an unserer Analyse, die ein Mixtum zwischen einer Rezension und einem selbständigen wissenschaftlichen Aufsatz darstellt, zeigt, wie schwierig eine künftige Edition von Dekretalensammlungen sein wird. Von 28 von uns erfassten Benedictus Levita-Stellen in den über 700 Kapiteln der Francofurtana kann er lediglich 5 Stücke ausfindig machen, die in direkter oder indirekter Beziehung zur Francofurtana stünden, also als ,fons materialis“ oder als „fons formalis“ zu bezeichnen wären. Das bedeutet, dass er nur einen einzigen zusätzlichen Text zu denjenigen beisteuert, die Landau bereits vor 18 Jahren veröffentlicht hat. Doch woher hat der Kompilator der Francofurtana diese Texte genommen? Wie Schmitz selbst schreibt, stammen sie nicht aus dem Werk des Benedictus Levita selbst. Er meint festgestellt zu haben, dass diese 5 in der Francofurtana rezipierten Texte in der Sammlung ,Abbreviatio Ansegisi et Benedicti Levitae', wovon fünf Handschriften aus dem 11. Jahrhundert stammen, ebenfalls enthalten sind. Dabei möchte er aber nur von einem „Verdacht" sprechen, dass „die Rezeption Benedicts [...] über die ,Abbreviatio ' gelaufen sein" könne. Und mit der ebenfalls unedierten, Collectio S. Hilarii Pictaviensis" als Vorlage der Francofurtana möchte er lediglich ,die Richtung benannt [...] haben, in der man weitersuchen müsste“. Diese beiden von Schmitz ins Spiel gebrachten Werke sind kaum weniger umfangreich als die Francofurtana. Es ist nicht einzusehen, warum mit ihnen die formale Quelle fuir die Benedictus-Levita-Stellen in der Francofurtana gefunden sein soll. Selbst Schmitz musste zu dieser Erkenntnis kommen!

1) Den englischen Einfluss auf Francofurtana $M$ und $R$ versucht Drossbach in ihrer in Druck befindlichen Analyse der englischen Sammlung Cheltenhamensis nachzuweisen. 
Obwohl wir meinen, dass Schmitz' Kritik an unserer Analyse letztlich auf einem methodischen Missverständnis beruht, sind wir ihm für zahlreiche Korrekturen, auf die wir hier im Einzelnen nicht noch einmal einzugehen brauchen, zu Dank verpflichtet. Richtigstellungen sachlicher Art werden gerne entgegengenommen, nicht aber Vorwürfe, eine Aufgabe nicht oder unzureichend erfüllt zu haben, wenn die Zielrichtung der wissenschaftlichen Arbeit der Autoren eine ganz andere war als die vom Rezensenten postulierte.

München

Gisela Drossbach

\section{Appendix von Peter Landau}

Im Folgenden möchte ich lediglich exemplarisch nur einige Punkte zur Kritik von Gerhard Schmitz aufzählen, um zu zeigen, dass er sich in der Bestimmung des fons materialis in der Francofurtana wiederholt irrt und folglich nach wie vor Francofurtana T der Urform derselben Sammlung am nächsten steht:

1. Die richtige Inskription der Dekretale von Frcf. 38.8 (S. 245) lautet „Januensi“, da der Adressat der Erzbischof von Genua war (vgl. Holt zmann, Kan. Erg. Nr. 114). Die Adresse „Ravennati“ taucht erst in jüngeren Dekretalensammlungen auf; ebenso in den Handschriften Francofurtana M und R als Korrektur. Die Inskription „Men.“ in $\mathrm{T}$ ist offenbar eine Verschreibung von „Januensi“, ebenso wie „Aven.“ in der Collectio Florianensis.

2. In Frcf. 38.11 (S. 247) ist nicht die Inskription in F, R, M gegenüber dem Urtext verändert, sondern der Schlusssatz. Er lautet im Urtext „Causa vero per appellationem ad primos delegatos aut ad eum, qui delegavit secundum appellantis arbitrium referetur". Die Handschriften F, R, M verändern delegavit zu appellaverit, was keinen Sinn ergibt, wobei $\mathrm{R}$ und $\mathrm{M}$ appellaverit nochmals zu delegaverit korrigieren. Die richtige Lesart des Schlusssatzes bringt nur T.

3. Bei Frcf. 56.15 (S. 350f.) ist in den Handschriften T, R, M die Rubrik von Frcf. 56.16 hierher unrichtig versetzt $-\mathrm{F}$ hat keine Rubrik. Hier bringt allein $\mathrm{T}$ ein Verweiszeichen auf Frcf. 56.16, was für eine höhere Bewertung von $T$ gegenüber $R$ und M spricht.

4. Die Handschrift $F$ muss jedenfalls deutschen Ursprungs sein, da sie bei Frcf. 1.9 „Francia“/Frankreich zu „Franconia“/Franken und bei Frcf. 3.2 sowie Frcf. 22.13 „Herefordensi“//Hereford zu „Herfordensi“//Herford verändert.

5. Bei Frcf. 7.2 (S. 66f.) ist c. 48 Canones apostolorum sicher fons materialis. Da das Kapitel weder bei Burchard noch bei Anselm oder Ivo rezipiert ist, bleibt eine direkte Benutzung Pseudo-Isidors wahrscheinlich.

6. Die Bemerkung über eine erweiterte Fassung des Textes in Ivos Dekret in Frcf. 16.9a (S.91) beruht darauf, dass nach der Edition von Migne, Ivo Decr. 5,165 nach deferat locum hinzufügt: „qui posterius ordinati sunt prioribus se non audeant anteferre".

7. In Frcf. 56.13 (S. 349f.) entsprechen die Hinweise auf Burch. 6,5 und Ivo Decr. 10,134 der jeweiligen Zählung in der Edition von Migne, Burch. 6,6 wiederholt den Text von Burch. 6,5 in etwas veränderter Form. Frcf. 56.13 übernimmt mit dem Ex- 